\title{
Integrated telemedicine applications and services for oncological positron emission tomography
}

\author{
GEORGE KONTAXAKIS ${ }^{1}$, DIMITRIS VISVIKIS ${ }^{2}$, ROLAND OHL ${ }^{3}$, ILIAS SACHPAZIDIS ${ }^{4}$, \\ JUAN PABLO SUAREZ ${ }^{5}$, PETER SELBY ${ }^{3}$, CATHERINE CHEZE-LE REST ${ }^{2}$, ANDRES SANTOS ${ }^{1}$, \\ FERNANDO ORTEGA ${ }^{6}$, JAVIER DIAZ $^{6}$, LEYUN PAN $^{7}$, LUDWIG STRAUSS $^{7}$, \\ ANTONIA DIMITRAKOPOULOU-STRAUSS ${ }^{7}$, GEORGIOS SAKAS ${ }^{3,4}$ and MIGUEL ANGEL POZO ${ }^{5,8}$ \\ ${ }^{1}$ Departamento Ingeniería Electrónica, ETSI Telecomunicación, Universidad Politécnica de Madrid, \\ Ciudad Universitaria s/n, 28040 Madrid, Spain; ${ }^{2}$ Centre Hospitalier Universitaire Morvan, 5 Avenue Foch, \\ 29200 Brest, France; ${ }^{3}$ MedCom Gesellschaft für medizinische Bildverarbeitung mbH, Rundeturmstr. 12; \\ ${ }^{4}$ Department of Cognitive Computing and Medical Imaging, Fraunhofer Institute for Computer Graphics, \\ Fraunhoferstr. 5, 64283 Darmstadt, Germany; ${ }^{5}$ Centro PET Complutense, 28040 Madrid; \\ ${ }^{6}$ Fundación Instituto Valenciano de Oncología, c/ Beltrán Báguena 8, 46009 Valencia, Spain; \\ ${ }^{7}$ Clinical Cooperation Unit Nuclear Medicine, Medical PET Group - Biological Imaging, \\ German Cancer Research Centre, Im Neuenheimer Feld 280, 69120 Heidelberg, Germany; \\ ${ }^{8}$ Instituto Pluridisciplinar, Universidad Complutense de Madrid, P ${ }^{\circ}$ Juan XXIII, n 1, 28040 Madrid, Spain
}

Received September 6, 2005; Accepted September 30, 2005

\begin{abstract}
TENPET (Trans European Network for Positron Emission Tomography) aims to evaluate the provision of integrated teleconsultation and intelligent computer supported cooperative work services for clinical positron emission tomography (PET) in Europe at its current stage, as it is a multi-centre project financially supported by the European Commission (Information Society, eTEN Program). It addresses technological challenges by linking PET Centres and developing supporting services that permit remote consultation between professionals in the field. The technological platform (CE-marked) runs on Win2000/NT/XP systems and incorporates advanced techniques for image visualization, analysis and fusion, as well as for interactive communication and message handling for off-line communications. Four PET Centres from Spain, France and Germany participate to the pilot system trials. The performance evaluation of the system is carried out via log files and userfilled questionnaires on the frequency of the teleconsultations,
\end{abstract}

Correspondence to: Dr George Kontaxakis, Departamento Ingeniería Electrónica, ETSI de Telecomunicación, Universidad Politécnica de Madrid, Ciudad Universitaria s/n, 28040 Madrid, Spain

E-mail: g.kontaxakis@upm.es

Key words: positron emission tomography, health telematics networks, computer supported collaborative work, tele-consultation, interface sharing, virtual private networks their duration and efficacy, quality of the images received, user satisfaction, as well as on privacy, ethical and security issues. TENPET promotes the co-operation and improved communication between PET practitioners that are miles away from their peers or on mobile units, offering options for second opinion and training and permitting physicians to remotely consult patient data if they are away from their centre. It is expected that TENPET will have a significant impact in the development of new skills by PET professionals and will support the establishment of peripheral PET units. To our knowledge, TENPET is the first telemedicine service specifically designed for oncological PET. This report presents the technical innovations incorporated in the TENPET platform and the initial pilot studies at real and diverse clinical environments in the field of oncology.

\section{Introduction}

Positron emission tomography (PET) is a molecular imaging technique, with the potential to yield the physiologic information necessary for oncological diagnosis based on increased regional metabolism of radio-labelled compounds and also to serve as a valuable tool for monitoring chemotherapeutic effects and the early diagnosis of tumour appearance and recurrence for diagnosis as well as treatment planning and management of patients with cancer, apart from its contributions to medical and pharmacological research (1).

PET is able to locate in quantitative way the regional distribution of specific compounds introduced in tracer quantities in the body of the patient, labelled with radioisotopes which decay emitting, among others, positrons. These particles annihilate with free electrons in the surrounding 
tissues producing a pair of $\gamma$ rays which travel in opposite directions at the speed of light. Current PET technology is able to detect both $\gamma$ generated, with extraordinary precision in terms of time of arrival of the rays to the detectors, with which the PET camera is equipped, and their energy. Based on this information, sophisticated processing and analysis of the information provided by all detected $\gamma$ ray pairs generates an accurate image on the distribution density of the tracer compound within the body, which can in addition provide valuable quantitative information on the process of the uptake of the tracer by the various organs and tissues.

Traditional diagnostic techniques, such as radiography, computerized tomography (CT) scans or magnetic resonance imaging (MRI), produce anatomical images of the internal organs. The premise with these techniques is that there is a visible structural change in anatomy caused by disease. However, biochemical processes are also altered with disease and may occur before there is a change in gross anatomy. Furthermore, PET can provide medical doctors with important early information on very subtle changes of function in tissues, due to disease-related modifications in tissue perfusion, cell metabolic rates, etc. This allows physicians to diagnose and treat these diseases earlier and consequently more efficiently and accurately, according to the axiom 'the earlier the diagnosis, the better chance for treatment'. PET can also help physicians monitor a patient's response to treatment, as well as identify distant metastases that can affect treatment, helping curtail ineffective treatments and reduce unnecessary invasive procedures. The field of PET is emerging into clinical diagnostic medicine and is approved by many insurance carriers for coverage.

Although complementary to other medical imaging modalities (MRI, CT, etc.), PET is often installed in independent health care centres (known as PET Centres) or in separate units within the nuclear medicine departments of major hospitals. The somehow autonomous nature of a PET Centre is due to its extremely specialised and expensive instrumental equipment and maintenance, the requirements for a particular construction for its housing, its high demand for financial input and needs for highly qualified and trained multidisciplinary personnel.

PET requires highly qualified physicians and technicians and only few PET Centres operate in each European country, separated by language and cultural barriers. Furthermore, some centres are specialised in particular cases whereas others count only little time of operation and consequently need support, training and advice from PET Centres with long-time experience.

TENPET comes to cover these needs, develop specific services for the technology implemented and set the first step towards the formation of a solid network covering most of the European PET sites. Moreover, the wider TENPET network includes some of the major referring centres (general hospitals, large health care centres, etc.) to the PET Centres, with the aim to facilitate the process of patient referral and allow the ordering physician to link and discuss directly with the examining physician the patient's images and results.

This project can be classified in what has been called telenuclear medicine by the Society of Nuclear Medicine, which has recently published a list of guidelines that outline the capabilities needed for a tele-nuclear medicine system (2). The major need for such a service has been identified to be remote reporting, especially in emergency cases (3). Javabased applications have been reported on the implementation of a tele-nuclear medicine system a few years ago (4), however activities in this field are scarce. Recently, a French group reported on the development of an electronic light-box called Positoscope (5), onto which PET studies can be downloaded, displayed, reported and sent to remote sites for expert advice. This device looks like a classical light-box equipped with a small touch-screen and a digital sound recorder. The system can be connected to local PET scanners and long distance high speed networks, with the primary purpose to transmit difficult PET studies to remote experts for evaluation.

The TENPET project goes beyond the restricted principle of remote reporting, by allowing physicians to conduct a real-time integrated on-line session on a specific case. At the same time provides an advanced platform for image visualization, analysis and processing, as well as a complete database system for data storage and autonomous operation within a clinic. In addition, it is the only system specifically available for PET that can link together the PET imaging centre and the referring physicians in a simple to use platform, which runs on conventional PC platforms and operates in the plug-and-play principle without long installation or training phases.

\section{Materials and methods}

System architecture. TENPET is based on the TeleConsult software platform, which is the successor of TeleInViVo (6), developed by Fraunhofer Institute for Computer Graphics (IGD), Darmstadt, Germany. Both platforms are medical imaging applications with telemedical and tele-collaborative functionality. Thereby TeleInViVo focused on the acquisition and transfer of sequences of ultrasound images in the context of health care for isolated areas such as islands, rural and crisis situation areas.

The TeleInViVo platform has been adapted and amplified, so that it can be used in general with all kinds of medical images, especially in appliance of the DICOM standard (7). In addition new functionalities, especially the transfer of messages and collaboration between doctors in two different modes (on-line and off-line) has been added. This platform released by MedCom $\mathrm{GmbH}$ in Darmstadt, Germany (8) has been adapted for the usage within the TENPET project, especially with the focus on visualization of PET images and the fusion of images from different modalities, which permits the overlay of CT and/or MRI image sets on the PET images for a better diagnosis and patient management. Moreover, considering the exigency of data security, especially when dealing with highly sensitive patient information, new technologies for data encryption and secured transmission have been added.

The TENPET platform is designed for Microsoft Windows NT/2000/XP. The main application is as an integrated tool for volumetric dataset image visualisation, analysis and processing, specifically designed and adapted to the needs of oncological PET studies. 


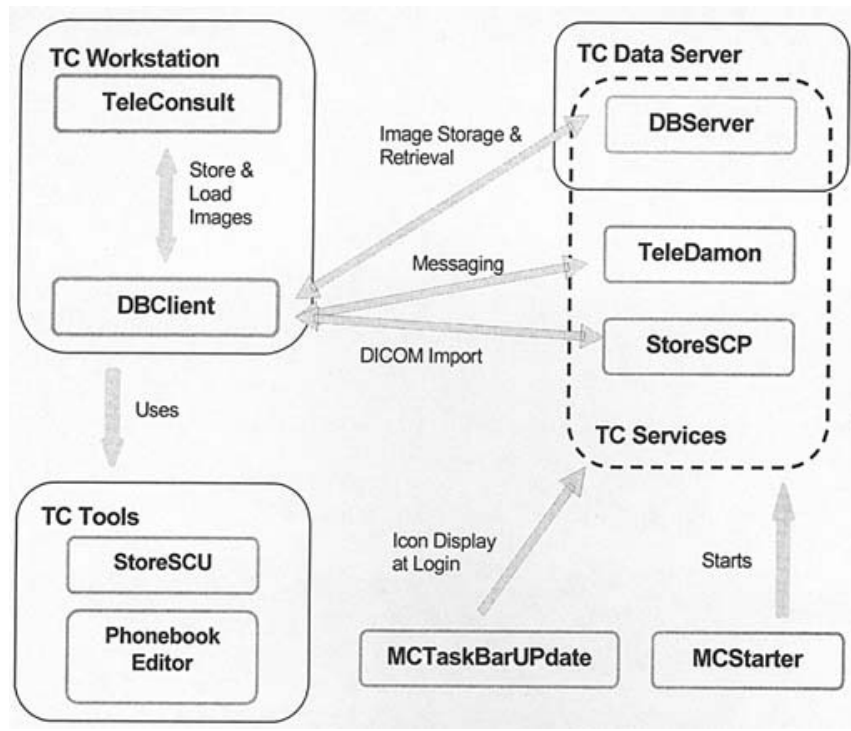

Figure 1. Overall system architecture.

It is composed by a series of modules that operate in synergy. Each module runs as an independent process on the PC. The principal modules are the following: a) the main application, a combination out of DICOM viewer, image grabbing software and medical telecommunication tool; b) a database client, which is a tool for the data access, both locally and in connection with a database server; c) a database server (DBServer), which makes possible the use of one single data pool, available for example to the whole clinic with one or several TeleConsult workstations; d) the telecommunication service (TeleDaemon), which is handling the exchange of messages with other TeleConsult workstations; e) an implementation of the DICOM Store Service Class Provider (SCP) service, which handles the receiving of images pushed from other DICOM devices; f) an implementation of the DICOM Store Service Class User (SCU) Service, which handles the transmission of files from TeleConsult to DICOM-supporting applications; g) an internal phonebook, where the communication partners of a TeleConsult workstation are stored; h) a starter of the TeleDaemon, DBServer and StoreSCP services, when booting a PC. In some cases these services run, even if no one is logged in to a workstation; i) a service that informs a user that logs in about the services that can display their icons in the windows task bar.

Fig. 1 gives an overview of the different modules that put together the TeleConsult platform. The two main applications that are running on a TeleConsult workstation are TeleConsult and the Database Client. Thereby TeleConsult is liable for the image visualization and the collaboration of doctors in the on-line mode. The Database Client is responsible for the storage of the data in the database, which is usually Microsoft Access based and for the sending of messages in the off-line mode. The usage of a data server is optional but recommendable, if TeleConsult is used in a clinical environment, where more than one TeleConsult workstation is used, but from each station, the same data basis would be available (Client/Server-Concept).
In addition, several services belong to the TeleConsult system. Thereby the applications StoreSCU and StoreSCP are responsible for providing the interface to other medical applications and devices using the DICOM protocol. StoreSCU is necessary for the sending of DICOM files according to the standard and the StoreSCP service handles incoming DICOM files from other devices and applications. The TeleDaemon is the tool, which handles the sending and the receiving of off-line-messages from one TeleConsult system to another.

Image visualization. The main challenge of the TeleConsult platform is the visualization of 3-dimensional PET studies. Thereby the images can be displayed in a 3-dimensional and in a 2-dimensional view. Internally the images are stored in a voxel cloud. Through projecting the voxels, cuts through all three planes (coronal, axial, saggital) can be visualized.

For the display of PET studies usually special color lookup tables are used. The images in Fig. 2 are visualized with an inversion LUT.

Image fusion and registration. An additional part of the visualization of TeleConsult is the fusion module. Under image fusion we understand the merging of images from different modalities (for example PET and CT or MRI) into one single volume (9). Therefore, a geometrical transformation of one volume into another is necessary. The geometrical transformation of a secondary volume is the result of a volume registration (10). This transformation is rigid. There is no scaling or distortion of the secondary volume that results from the volume registration. The transformation is represented using the following parameters: a) $x-y-z$ shift of the referencepoint or iso-centre based registration; b) $\mathrm{x}-\mathrm{y}-\mathrm{z}$ shift of manual, landmark based, VOI based and automatic registration; c) $\mathrm{x}-\mathrm{y}-\mathrm{z}$ rotation of manual, landmark based, VOI based and automatic registration; for further processing these parameters are used to build up a homogeneous $4 \times 4$ matrix $\mathrm{M}_{\mathrm{ij}}$ :

$$
M i j=\left[\begin{array}{cccc}
i_{0} j_{0} & i_{0} j_{1} & i_{0} j_{2} & i_{0} j_{3} \\
i_{1} j_{0} & i_{1} j_{1} & i_{1} j_{2} & i_{1} j_{3} \\
i_{2} j_{0} & i_{2} j_{1} & i_{2} j_{2} & i_{2} j_{3} \\
0 & 0 & 0 & 1
\end{array}\right]
$$

Volume shifts are movements of the secondary volume in millimeters. The maximum accuracy of volume shifts is $0.1 \mathrm{~mm}$.

The $x-y-z$ directions of the shift are not defined according to the patient's position and orientation but to the user's view onto the orthogonal cuts of the primary volume. A shift value is given as the distance between the centers of the corresponding volumes (Fig. 3).

Volume rotations are defined as rotations around the $\mathrm{x}-$, $y$ - and $z$-axis of the shifted secondary volume. They are given in degrees with a maximum accuracy of 0.1 degree. If a registration consists of rotations around more than one axis, the secondary volume is rotated around its axis in the following order: a) rotation around $\mathrm{x}$-axis; b) rotation around $\mathrm{y}$-axis; c) rotation around z-axis. 

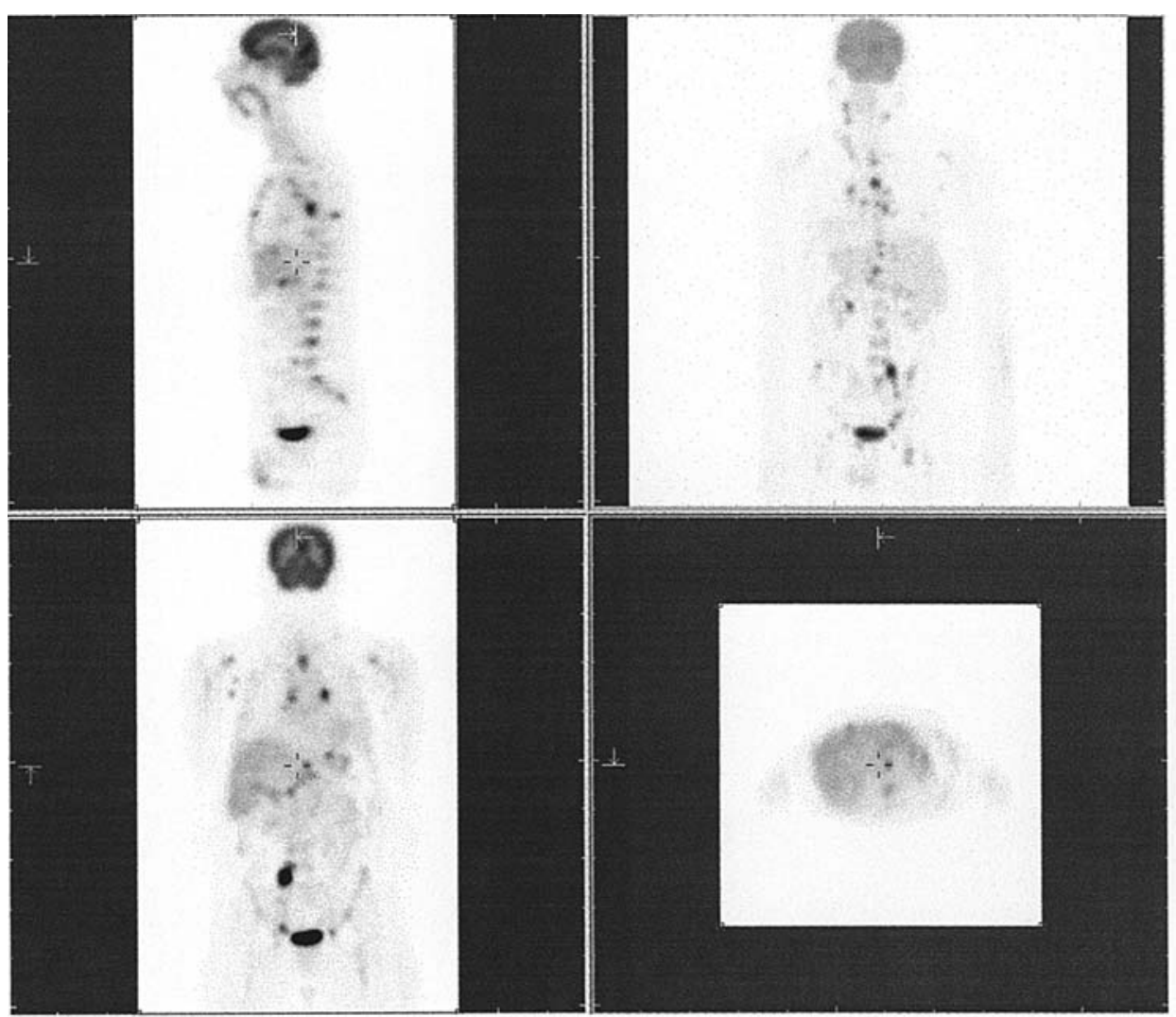

Figure 2. Whole-body-PET-study displayed with TeleConsult.

Thereby the transformed volume is the secondary volume and the volume with fixed location and rotation is the primary volume. Within TeleConsult there are three different techniques of registration implemented: a) manual registration; b) marker-based registration; c) automatic registration.

The manual registration allows rotation and shifting the secondary volume manually. It can be applied either by using the corresponding sliders in the user interface or by dragging the mouse pointer in the slice windows of the fused volumes.

The marker-based registration (landmark-based registration) allows the definition of landmarks that can be used to register two corresponding volumes. The number of landmarks is not limited and can lie in the interval:

$$
N_{\text {Landmarks }} \in[0, \infty)
$$

The landmarks are stored in separate lists for the primary and the secondary volume. The maximum number of landmarks is only limited by the available memory. Only corresponding landmarks (called landmark pair) can be used for the registration. Landmark pairs are landmarks in both volumes that share the same marker name.

The automatic registration method allows an automatic calculation of the transformation for the secondary volume. Bounding boxes can be applied for the primary and the secondary volume and it is possible to choose whether to speed-up the process or to calculate the registration result with a higher precision. The automatic registration algorithm uses the grey values of the secondary and the primary

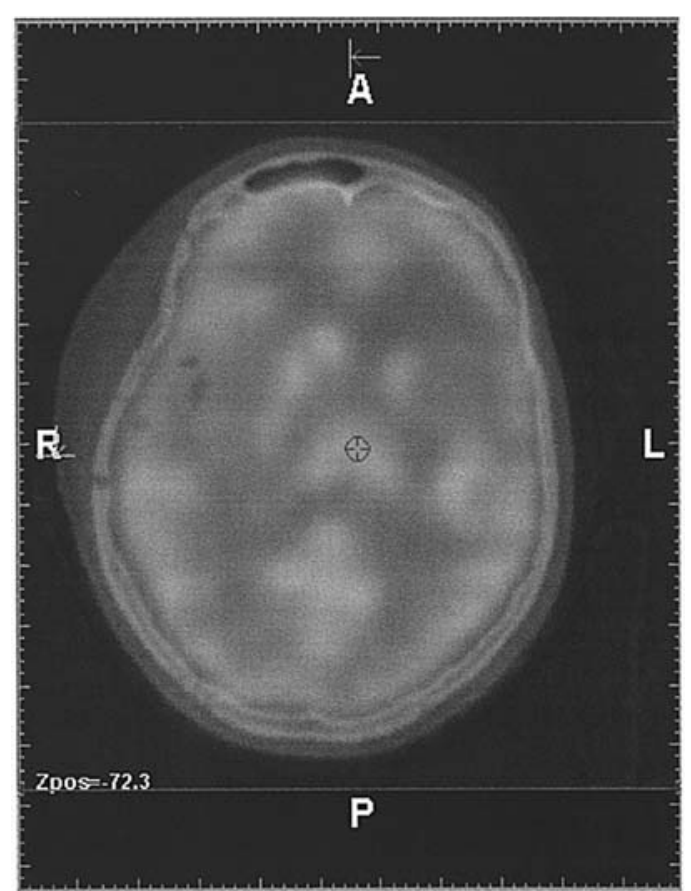

Figure 3. Image fusion with PET and CT images.

volume and a mutual information approach to determine if a transformation moves the secondary volume correctly according to the primary volume. The mutual information approach allows registering the volumes independently of the volume modalities (11). 


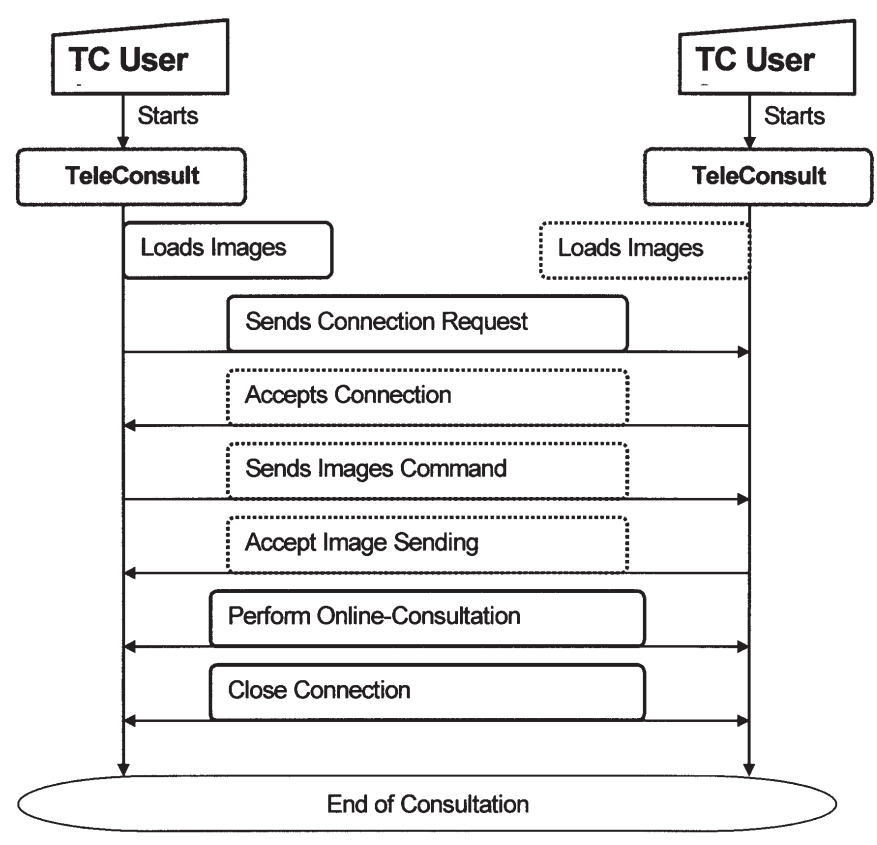

Figure 4. Workflow of an on-line teleconsultation.

Telemedical collaboration. Apart from the PET image visualization and the fusion with images from other modalities, the main target of TeleConsult is, to increase the quality of patient treatment and scientific work in the way of making the collaboration between two doctors or institutes easier. Thereby we focus on the sending of images, together with additional useful information from one place to another and on the discussion of these images on-line, whereby the discussion partners are in different places.

Thereby we distinguish between on-line collaboration and off-line messaging. For the on-line collaboration it is necessary that both users are on-line at the same time, whereby off-line messaging does not require that the remote communication partner is available, when the messages are sent.

Both methods have their advantages and disadvantages. On-line sessions have more options for the interaction and exchange of information. Especially within TeleConsult the principle 'what you see is what I see' (WYSIWIS), where both partners have the same view of the images during the on-line-session, has great advantages. The main disadvantage is that both communication partners need to be available at the same time, something that is not necessary in the case of the off-line messaging method.

The on-line communication between two workstations is implemented over TCP/IP based point-to-point connections. Fig. 4 shows the usual workflow of a tele-collaboration session. After both partners have connected, the first step is to exchange the images. This step is optional, because it is possible to send the images in advance by using the off-line messaging functionality. Images are therefore only transmitted if they do not already exist in the database of the remote communication partner. During the step 'perform on-line-consultation' a wide range of manipulations of the visual images is possible. In addition, the two doctors can communicate (chat) and add additional graphical and textual annotations to the images, in order to render more precisely their conclusions. For the synchronisation of the two connected applications, only short commands are sent. This enables on-line consultations even over connections with a low bandwidth, using the principle of interface sharing.

Off-line messages are usually sent in the absence of the receiver of the message. The off-line messaging functionality is part of the database client user interface. The TeleDaemon that is running on the server in the remote clinic is the handling of the received messages. Off-line messages can contain in addition to the images, additional information to the images (annotations), textual and audio messages from the sender and a wide range of other documents and files. This means that the messages are similar to an e-mail, but the images are automatically stored in the database of the receiver. Fig. 5 gives an overview of the elements of the on-line and off-line tele-communication.

Image compression. The issue of the transmission of large data sets over the (usually low) bandwidth had to be solved. In addition to the possibility of off-line-sending, for the on-linesending a wavelet-based image compression algorithm (12) has been implemented for the size reduction of the image data sets to be transmitted. The compression is lossy, but very efficient in cases where bandwidth is a crucial issue. Otherwise, a loss-less compression can be used for the data transmission. In addition, the available image formats, e.g. DICOM have already the possibility to store images in a compressed format.

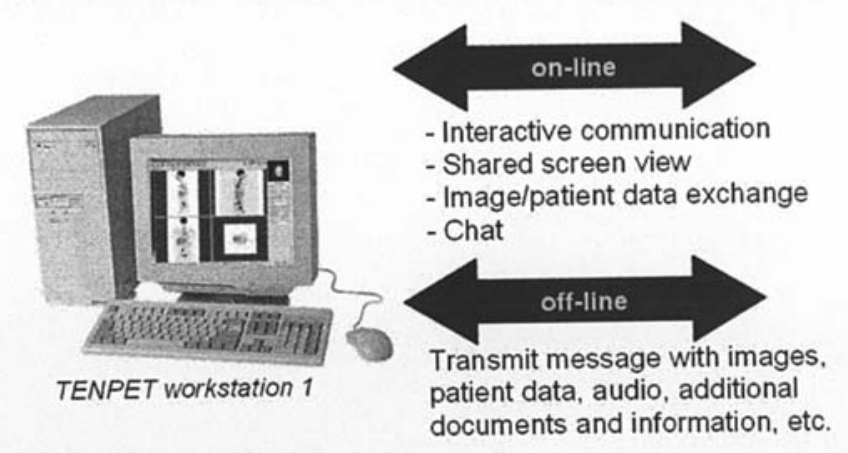

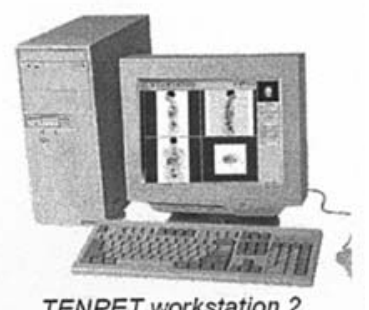

TENPET workstation 2

Figure 5. Elements of the TENPET communication modes. 


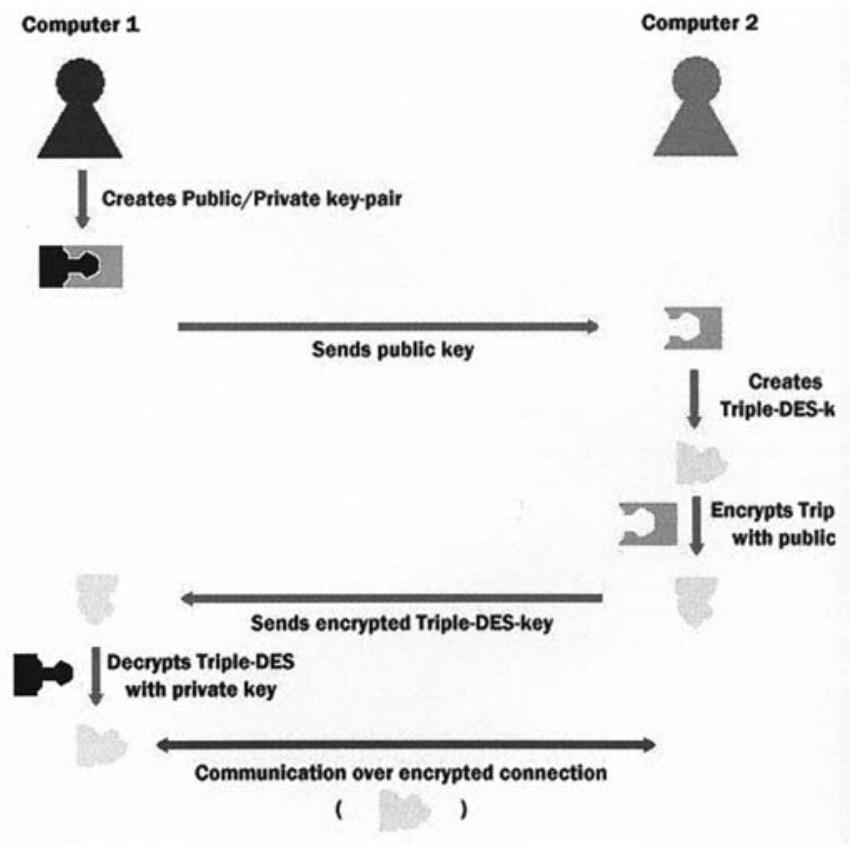

Figure 6. Encrypted connection.

Security issues. Modern local area networks that are standard for research institutes and larger hospitals bring new challenges for CSCW-software. Techniques like firewalls and network address translation are protecting the network. This kind of protection was not relevant for the TeleInViVo project, but must be considered for the newer applications. In addition, the sensitive patient information must be protected, considering the requirements of data protection.

Within the TENPET project this last issue has been solved with the automatic de-personalization of patient information before transferring the images and through establishing encrypted connections. Thereby we use a combination of public-key/private-key and Triple-DES encryption (Fig. 6).
The main problem when using firewalls is that usually point-to-point connections will not work, because firewalls are blocking incoming connection requests. Therefore, it is necessary to have a solution, which is working server-based, and where the connection between two TeleConsult workstations is always mediated through a server. To solve this problem, a virtual private network (VPN) has been established for all users within the project. The communication is then handled in two steps.

First the TeleConsult workstation connects to a VPN concentrator and logs in to the TENPET virtual private network. Afterwards the users can establish point-to-point connection without being blocked by the firewall. In addition, because the virtual private network is established using SSL technology, this increases also the security of the data transmission (Fig. 7).

The use of VPN however implies several restrictions, like the necessity that even for off-line transmissions both workstations must be on and connected to the network. Furthermore, the user each time starts the application must in addition $\log$ in to the VPN (although this action is performed automatically at start-up). The use of the jabber protocol is being implemented and will be replacing the VPN in the near future.

Jabber is an instant messaging system such as MSN, ICQ or AOL. Instant messaging systems (13) are considered a convenient way of chatting. Instant messaging is used for business to business communication $(14,15)$. In the future, instant messaging system will gain ground and will be deployed in many applications such as medical applications (16). Within our project, we integrated an instant messaging system taking advantage of the jabber protocol.

Jabber instant messaging system is different from other instant messaging systems since it is based on XML. In addition, jabber is an open source XML protocol, which makes jabber more distributed and easily accessible independent of the platform.

A jabber instant messaging system consists of a hub server and many remote nodes, which are able to be connected to the

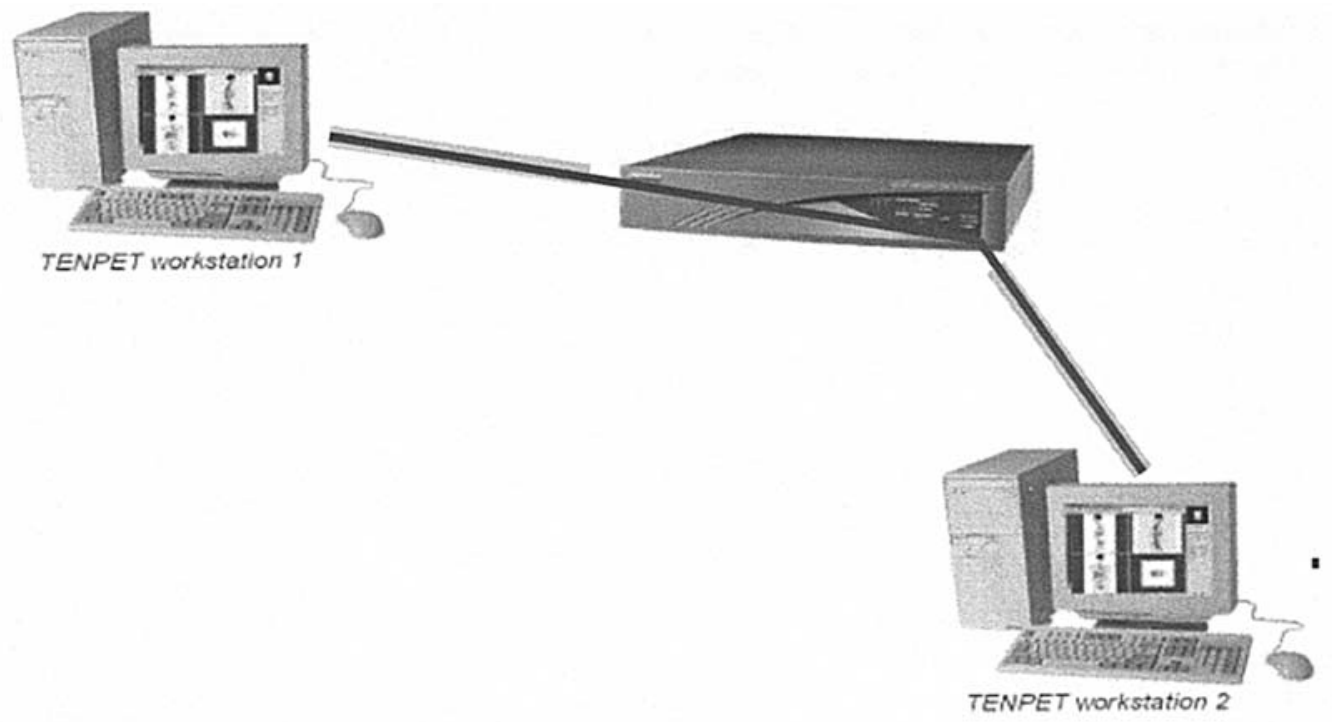

Figure 7. VPN-tunnel point-to-point connection. 


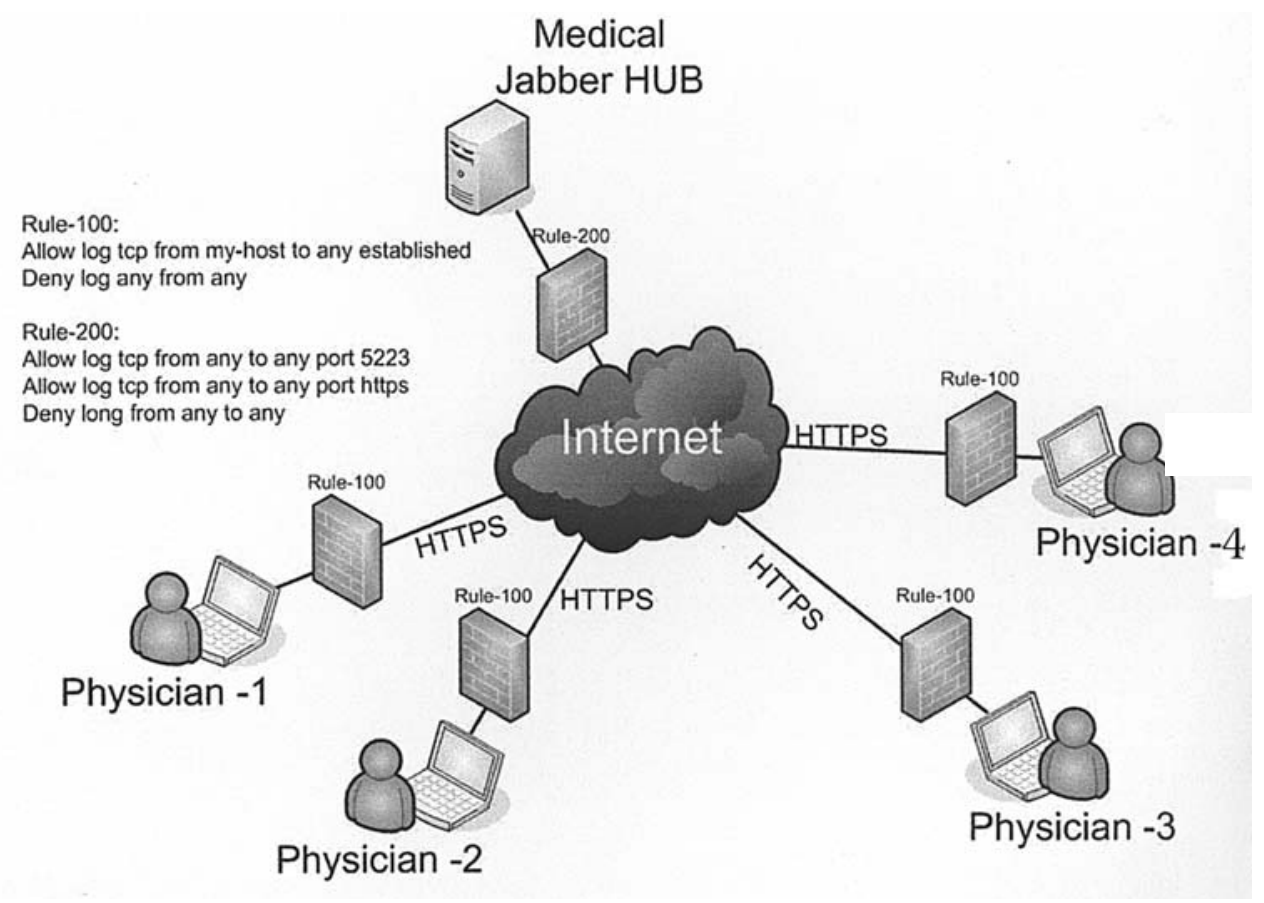

Figure 8. Jabber network

server. The hub is responsible for keeping track of user presence and to forward the messages to the correct user.

Jabber protocol (17) can be easily encapsulated into HTTP and be forwarded through firewalls. A very common problem among medical applications is how two different remote applications could exchange information when they are located behind firewalls, which permit only outgoing traffic. We were able to tackle this problem by placing a server somewhere in the Internet, which accepts incoming connections, as shown in Fig. 8.

Additionally, jabber protocol supports data transmission over HTTPS and SOCKS5 proxies. Furthermore, for users working in very restricted environments, with very tight firewall rules, jabber offers a polling connection method over secure HTTP (HTTPS).

Comparing the jabber instant messaging system to a VPN-based system we have to denote that jabber is more flexible when only http and https protocol are available. At the same time jabber is as secure as VPN since SSL is used in conjunction with HTTP.

Additionally, using jabber protocol the medical application does not need to keep track of the user presence. Jabber server is responsible for this function. The application at all times is aware of the presence of the register users. In this way, we support the mobility of the doctors. They are completely free to work at any available terminal or even when they are moving around.

\section{Results}

The platform is currently in clinical trials, which started in February 2005 and are expected to last until December 2005. A number of telecommunication sessions between the participating centres have been already carried out, demonstrating a time-efficient and secure approach in sharing experience for the interpretation of PET cases. In addition, during remote reporting sessions excellent ease of use was reported in $>80 \%$ of the cases. Following is a detailed description of the TENPET trial scenarios at each of the participating clinical PET sites.

Centro PET Complutense, Madrid, Spain (CPC). Upon the initiation of the medical trials $\mathrm{CPC}$ holds regular weekly sessions with the PET unit at the Fundación Instituto Valenciano de Oncología (FIVO), the second participating PET trial site in Spain. One nuclear medicine physician is involved from each of the two centres. One or two patient cases are transmitted by FIVO and discussed either on-line or off-line during each session. A total of about 30-35 teleconsultation sessions are expected to take place. For the situation of an urgent case that calls for an out-of-schedule transmission of data, off-line transmissions are used and the patient data are available for examination by the PETMAD physician at his earliest convenience.

$\mathrm{CPC}$ is also evaluating the system for remote reporting during the absence of its leading nuclear medicine physician, when he attends international academic conferences, lectures or other events related to molecular imaging. During those periods, the medical doctor has the chance to access the data of the daily patient examinations, review them using the TENPET system and issue the reports from a remote location.

Furthermore, CPC also evaluates the TENPET system in the scenario of patient data transmissions between the PET Centre and a peripheral hospital, which refer patients for PET examinations. This hospital uses the TENPET platform to send patient MR images to CPC, which will perform the PET study, issues the diagnostic report and returns to the hospital 
this report along with the PET data. One physician from each site is involved in these trials. It is expected that a total of 810 sessions will be held with the external referring centre until the end of the trial period.

The Centro PET Complutense holds also teleconsultation sessions with the German Cancer Research Centre (DKFZ). CPC transmits to the group at DKFZ difficult oncological cases (dynamic ${ }^{18} \mathrm{~F}$-FGD PET studies, on the analysis of which the DKFZ group has a long-year experience) for discussion and second opinion. Two physicians from CPC and two physicians from DKFZ are involved in these sessions.

Centre Hospitalier Universitaire Morvan, Université de Brest Occidentale, Brest, France (UBO). Based on the PET workload within the Nuclear Medicine department of the Centre Hospitalier Universitaire de Brest (CHU) there are on average 25-30 examinations per week. Of these about 5$10 \%$ are estimated to be eligible, due to their complications in diagnosis and interpretation, for remote reporting using TENPET services.

A total of 15 examinations per month are planned to be sent via the TENPET system for a second opinion. This activity will take place between the different collaborating centres of the project, mainly the experienced PET Centres in Spain (CPC) and Germany (DKFZ). Two nuclear medicine physicians will be involved in this task.

Furthermore, the TENPET software has been installed in the laptop of the leading nuclear medicine physician at UBO. Remote reporting sessions are performed when she is not present at the hospital premises, either during out-of-office times or when she is away on a trip.

UBO will also provide a preliminary feasibility study on other utilisation models of the TENPET platform by installing the system within the department of radiotherapy. There is currently an increased interest in the use of PET for radiotherapy treatment planning (RTP) and within the UBO there are projects associated with tumour delineation in PET images for use in RTP.

Furthermore, UBO makes use of the TENPET platform as advanced image visualisation software to be used at interdisciplinary meetings within the hospital or at other meetings outside, during case study discussions, seminar or workshop presentations, as well as in the training and education of students at the Laboratoire de Traitement de l'Information Médicale (LATIM) which closely collaborates with the nuclear medicine staff as research and technology support partner.

Fundación Instituto Valenciano de Oncología, Valencia, Spain (FIVO). Since the initiation of the medical trials FIVO holds regular weekly sessions with the Centro PET Complutense, the Spanish PET Centre of Excellence participating to the project, according to the schedule shown above.

Deutsches Krebsforschungszentrum, Heidelberg, Germany (DKFZ). The Medical PET Group - Biological Imaging at the German Cancer Research Centre is focussing on the integration of software for the evaluation of PET images to support medical diagnostics and therapy management in oncological patients.
Research studies are performed with external partners at the local clinics in the area of Heidelberg as well as with partners in Greece and Israel. Within the current research projects, the studies with receptor active radiopharmaceuticals gain primary attention. ${ }^{68} \mathrm{Ga}$-DOTATOC is used in patients with endocrine tumours to achieve an accurate diagnosis. Furthermore, based on the quantitative data, the patient is referred to the Division of Nuclear Medicine at the University Clinic for treatment with ${ }^{90}$ Y-DOTATOC. Follow-up examinations are performed prior to each therapeutic cycle. The qualitative and quantitative data of the PET examinations are discussed with the referring physicians, e.g. from the University of Crete, Greece, and with the physicians responsible for the radioisotope treatment at the Division of Nuclear Medicine at the University of Heidelberg.

Another study of prime interest is performed in patients with colorectal tumours. PET studies are performed with ${ }^{18} \mathrm{~F}$-deoxyglucose (FDG) immediately prior to surgery for staging purposes. Following treatment, tumour specimens are evaluated with gene chip technology and gene expression data are compared to the quantitative PET results. This study is performed in cooperation with the Surgical Clinic at the Klinikum Ludwigshafen.

The TENPET software provides also the possibility to do a correlative analysis of PET data. The DKFZ team is focusing especially on the assessment of the image fusion module. Image fusion will be tested with PET data and CT as well as MRI images. The results will be compared to the fusion data obtained with PMOD (18), which is the standard software for PET data analysis used by the DKFZ physicians involved in the project.

\section{Discussion}

TENPET networks are being set up and validated in terms of health care and cost improvements, at both national (Spain) and European (Germany, France, Spain) levels. Their organization is based on the current procedures and protocols used by the involved health care providers, PET Centres and hospitals. Furthermore, these health telematics networks are based on the requirements of each user and are set up according to national and EU regulations and restrictions.

The expected users of the TENPET services are PET Centres, regional health centres, large and small hospitals and clinics, with or without nuclear medicine services, mobile PET units and the referring physicians. In the course of this project, the market validation of the TENPET platform, customers of the TENPET services are considered all health units and professionals related with PET imaging.

The TeleConsult platform, which implements the TENPET services, eliminates this hassle and offers to PET specialists a concrete tool and specific methodology to contact other colleagues and request their cooperation in an efficient, easyto-use and standardised procedure. Furthermore, both ends can exchange chat messages, send files (like medical reports, etc.) to each other, point regions of interest to the other partner with the telepointer, etc.

The major service categories for which there is a significant market margin for further development and business opportunity for the TENPET platform and services have been identified as the following ones. 
Second opinion. In this case a PET centre which is either new or is lacking experience will send the data to the more advanced centre for a second review. The problems herewith are diverse: a) second opinion in other centres is not a paid service by European Union law. This can be practicable only for areas where private funding is possible. This could be implemented mostly in Southern European countries; b) different protocols require different evaluation and thus a study replication; c) the doctors feel uncomfortable to ask for advice from another doctor who is considered to be an expert.

Concluding, this second opinion seems to be rather problematic. Possibly there could be a much better opportunity overseas. However, even in Europe this can be regarded as being a potential market especially for new-established PET Centres asking for a limited period of time the assistance and support of the partner centre.

In fact, this has happened in the past on several occasions and continues to happen now that many new PET sites open every year outside main capitals of the European countries. Even the Centro PET Complutense, coordinator of the TENPET initiative, at its early stage of operation in the mid 1990s had a support agreement with the University of California - Los Angeles (UCLA) for second opinion support on difficult cases.

Remote reporting. Remote reporting is linked to the previous case. It can also be extended to the case of mobile PET, where systems are mounted on trucks and are shared among peripheral hospitals. These peripheral hospitals perform the examinations of the patients in situ and send the data to the central expertise unit (Centre of Excellence) for evaluation. In addition to this, one PET scanner can be used as shared facility by several user groups, as it is a common case in France. Again, the data acquisition is taking place in one location and the evaluations of the data are performed in different sites.

In the remote reporting case, physicians have to report PET studies for which they feel they have a limited experience in some cases. By requesting remote expert advice, they get trained at the same time. At one point in time, they may feel well trained and request less expertise or the expert may judge the burden of performing expertise too time-consuming. In either case, the number of requests will decrease and the need for a TENPET will be questionable.

Another way to consider the future of the remote reporting feature would be to decide that data acquisition for complex imaging studies need not be performed at the same location as reporting depending where expertise is located. TENPET would then become a very user-friendly teleradiology device. Up to now teleradiology has been essentially considered for providing remote areas with high quality medical services. In the case of PET, teleradiology can be extended to situations where a radiologist or a nuclear medicine physician can perform the acquisition and the reporting of standard cases but can request the help of a remote expert when needed. One can even consider a network of radiologists or nuclear medicine physicians, each of them specialized in rare or complex imaging studies. In the latter case, acquisition of such studies could be performed all over the network while reporting would be performed by one or two members of the network. Such a network would also be a solution for ensuring that complex studies can be always reported, provided that experts of a given study are somewhere available at the time of the request.

Interdisciplinary communication. In this case, doctors from various specializations (diagnosis, surgery, radio-oncology) have to meet to discuss the case regarding the patient treatment. Such a discussion takes place in a physical room with all experts sitting in front of a whiteboard, light-box, etc. A potential application of telemedicine could be as a tool for virtual meetings among such specialists. In such a case, the technology should be extended by a video-conferencing and a speaker phone over internet capability.

The TENPET platform can be also used as a display of selected images of a given patient during multidisciplinary staff meetings. In this case, images are selected from various modalities and displayed on the monitor such that they can be seen by a large audience, and that the relationship between the various images can be understood easily by the audience.

Electronic fax. Here the system is used as a transportation medium for transferring the results of hospital back to the referring position. Currently this is done by courier services and films however this practice is becoming more and more difficult because the data are practically all in digital form. Electronic fax to referring positions can be a practicable solution only if the installation and the use is as simple as possible, but the location of the referring physician. Referring physicians are not willing to invest time and efforts in using a complicated system, anything being more complicated than a fax is already too complicated.

Advanced visualization console. Apart from the telemedical features integrated in the system, the TENPET platform could be of value itself if it offers features not available to the commercial scanners. Today the evaluation software accompanying the system is rather rudimentary and is not offering a lot of reviewing possibilities. However, this console is given 'for free' as a gift to the doctor purchasing the PET scanner. In order for this doctor to invest money on a different technology, the investment must be moderate and must offer significant benefits as compared to the software available to him today. Advanced fusion changing of colour look-uptables, segmentation and multislicing capabilities could be regarded as being such possibilities. The telecommunication features are additional to this.

Through the provision of a bundle of services via an easyto-use user interface, TENPET offers numerous benefits: a) allows the speedy, accurate and filmless communication between referring physicians and specialized medical personnel at a PET Centre; b) allows PET physicians to easily access the radiology data (CT, MRI, etc.) of the patient in digital form, ready to be registered and fused with the PET images for the final diagnosis. Until now, the common practice has been that PET reports are compiled based on CT or MRI films that come with the patient's records, viewed on the classic light-box; c) allows the exchange of experience between physicians and medical personnel from PET Centres with 
distinct specialisations, for diagnostic, training or scientific purposes; d) brings all PET Centres in Europe (and beyond) closer, breaking barriers of frontiers, language and culture nurturing the vision of many towards the foundation of the European PET Association; e) brings together advanced medical imaging technology and health telematics network applications to a platform that can be directly applied to the whole spectrum of teleradiology, thus increasing by orders of magnitude the market size of the developed services.

Within the broader context of TENPET, all countries are experiencing increasing demand for healthcare services and therefore, by implication, technology-driven diagnostic and screening programs and alternative methods of healthcare delivery are featuring in current policies and practice. Due to the increasing necessity and importance of healthcare in the community, there is a need for novel state-of-the-art systems and services that are compact, portable, interoperable and competitively priced. Healthcare industry professionals are considered to have an important part to play in the development of products and services in the environment of changing social and economic conditions. These can be considered to be the following: a) to be able to influence the development of new approaches to healthcare delivery and impact on the advancement of clinical practice; b) to contribute to the broadening of European healthcare industry influence and image, through implementation of practical solutions; c) create an environment for successful expansion into new business segments; d) outline product concepts that could be developed to satisfy the potential demand for remote care systems.

Furthermore, TENPET fosters and promotes the development of new target applications in the teleconsultation, networking infrastructures and architectures including the integration of fixed, mobile, on-line and off-line technologies and their application to advanced health telematics networks. The TENPET service is based on proven technology, taking into account recent advances in medical image technology, ubiquitous communication and intelligent interfaces, and is based on innovative systems, user-friendliness, cost effectiveness and quality of service.

In conclusion, TENPET promotes the cooperation and improved communication between PET oncologists, offering options for second opinion and training. Furthermore, it permits physicians to remotely consult patient data if they are away from their centre or work on mobile units. Through the provision of a bundle of services via an easy-to-use user interface, TENPET allows the speedy, accurate and film-less communication between referring oncologists and specialized medical personnel at a PET Centre. Furthermore, it brings all PET Centres in Europe (and beyond) closer, breaking barriers of frontiers, language or culture, by integrating advanced medical imaging technology and health telematics network applications into a single, easy to operate telemedicine platform. It is expected that when fully deployed, TENPET will have a significant impact in the development of new skills by PET professionals and will support the establishment of peripheral PET units.

\section{Acknowledgements}

TENPET is being partially funded by the Commission of the European Communities (DG Information Society, eTEN programme, contract Nr. C510711). Web site: http://www. tenpet.com

\section{References}

1. Strauss L: Positron emission tomography: current role for diagnosis and therapy monitoring in oncology. Oncologist 2: 381-388, 1997.

2. Parker JA, Wallis JW, Jadvar H, et al: Procedure guideline for telenuclear medicine 1.0. J Nucl Med 43: 1410-1413, 2002.

3. Parker JA and Wallis JW: Systems for remote interpretation of emergency studies. Semin Nucl Med 23: 324-330, 2003.

4. Slomka PJ, Elliott E and Driedger AA: Java-based remote viewing and processing of nuclear medicine images: toward 'the imaging department without walls'. J Nucl Med 41: 111-118, 2000 .

5. Bizais Y, Guédonc JP, Couturier O, et al: The POSITOSCOPE: an easy-to-use, communicating electronic lightbox. In: Medical Imaging 2004: PACS and Imaging Informatics. Ratib OM and Huang HK (eds). Proceedings of the SPIE 5371: pp140-151, 2004.

6. TeleInViVo project: http://www.igd.fhg.de/teleinvivo

7. Digital Imaging and Communications in Medicine (DICOM): http://medical.nema.org/

8. Homepage of MedCom GmbH: http://www.medcom-online.de

9. Haller JW and Caplan J: Medical Image Fusion, http://www. radiology.uiowa.edu/NEWS/imagefusion.html

10. Firle E, Chen W and Wesarg S: Registration of 3D U/S and CT images of the prostate. In: Computer Assisted Radiology and Surgery 2002. Proceedings. Lemke Heinz U (ed). Springer Verlag, Berlin, pp527-532, 2002.

11. Gomez Zardain J: Automatic Registration of PET and CT resp. PET and MRT Volume Data. Darmstadt, Technical University, Master Thesis, 2002.

12. Villasenor J, Belzer B and Liao J: Wavelet filter evaluation for image compression. IEEE Trans Imag Proc 2: 1053-1060, 1995.

13. Debbabi M and Rahman M: The war of presence and instant messaging: right protocols and APIs. In: Proceedings, First IEEE Consumer Communications and Networking Conference. pp341-346, 2004.

14. Gao J, Modak M and Dornadula S: Mobile Jabber IM: a wirelessbased text chatting system. In: Proceedings, IEEE International Conference on e-Commerce Technology. pp337-341, 2004.

15. Saito K: Maintaining trust in peer-to-peer barter relationships. In: Proceedings, 2004 International Symposium on Applications and the Internet (SAINT) Workshop. pp582-588, 2004.

16. Sachpazidis I, Ohl R and Sakas G: Instant messaging: a communication layer for medical applications. In: Proceedings of the 2nd Cairo International Biomedical Engineering Conference, 2004.

17. Web server of jabber foundation: http://www.jabber.org

18. PMOD: http://www.pmod.com 